\title{
Sub-concussive Hit Characteristics Predict Deviant Brain Metabolism in Football Athletes
}

\author{
Victoria N. Poole ${ }^{1,2}$, Evan L. Breedlove ${ }^{3}$, Trey E. Shenk ${ }^{4}$, Kausar Abbas ${ }^{4}$, Meghan E. \\ Robinson, $\mathrm{PhD}^{1}$, Larry J. Leverenz, $\mathrm{PhD}^{5}$, Eric A. Nauman, $\mathrm{PhD}^{1,2,3}$, Ulrike Dydak, \\ $\mathrm{PhD}^{6,7}$, Thomas M. Talavage, $\mathrm{PhD}^{1,4}$
}

\footnotetext{
${ }^{1}$ Weldon School of Biomedical Engineering, Purdue University, West Lafayette, IN 47907

${ }^{2}$ Department of Basic Medical Sciences, Purdue University, West Lafayette, IN 47907

${ }^{3}$ School of Mechanical Engineering, Purdue University, West Lafayette, IN 47907

${ }^{4}$ School of Electrical and Computer Engineering, Purdue University, West Lafayette, IN 47907

${ }^{5}$ Department of Health and Kinesiology, Purdue University, West Lafayette, IN 47907

${ }^{6}$ School of Health Sciences, Purdue University, West Lafayette, IN 47907

${ }^{7}$ Department of Radiology and Imaging Sciences, Indiana University School of Medicine, Indianapolis, IN 46202
}

\author{
Corresponding Author: \\ Victoria N. Poole \\ 150 S. Huntington Ave. \\ Boston, MA 02130 \\ Tel: (708) 539-5788 \\ e-mail:victorianpoole@gmail.com
}

This is the author's manuscript of the article published in final edited form as:

Poole, V. N., Breedlove, E. L., Shenk, T. E., Abbas, K., Robinson, M. E., Leverenz, L. J., ... \& Talavage, T. M. (2015). Sub-concussive hit characteristics predict deviant brain metabolism in football athletes. Developmental neuropsychology, 40(1), 12-17. http://dx.doi.org/10.1080/87565641.2014.984810 


\section{Introduction}

Understanding that neural injuries could have long-term detrimental consequences, there is increased pressure upon athletic directors, trainers, and coaches to determine the appropriate window of recovery for proper concussion management (May, Marshall, Burns, Popoli, \& Polikandriotis, 2014). Recent proton $\left({ }^{1} \mathrm{H}\right)$ magnetic resonance spectroscopy (MRS) studies argue that even the recommended two-week window of rest is insufficient for full metabolic recovery after concussion (Stefano Signoretti, Tavazzi, Lazzarino, \& Vagnozzi, 2014). Those returning to play prior to full recovery could incur a second concussion with symptoms and metabolic changes more lasting than the first (Vagnozzi et al., 2008) . These findings strongly suggest an additive effect of injury during a time of vulnerability. An additive effect of injury may also affect non-diagnosed players remaining in play, as supported by a recent ${ }^{1} \mathrm{H}$ MRS study (Poole et al., 2014).

This study seeks to determine whether head collision histories (e.g., number, magnitude, helmet location) can serve as meaningful predictors of sub-concussive changes in brain metabolism for athletes in play, and whether the accumulation of these hits can lead to more chronic injuries.

\section{Methods}

\section{Subjects}

Twenty-five male athletes $(M=16.6$ years; $S D=0.93)$ from the football teams at two high schools (Team 1: 14; Team 2: 11) participated over two years of study. No 
subjects were diagnosed by their athletic trainer or team physician as having been concussed during the study period.

\section{Subject Monitoring}

In each season, athletes participated in one assessment session before the beginning of contact practices (Pre-season) and at least once during the competition season with In-season sessions repeating 4-6 weeks apart $(M=36.2$ days, $S D=11.95)$. All athletes were also monitored for head collision events using the Head Impact Telemetry System (HITS; Simbex; Lebanon, NH), a sideline data collection system that reports the time, location, and magnitude of head impacts experienced by athletes. The HIT System has been employed in a number of studies of football-related neurotrauma (Broglio et al., 2010; Crisco et al., 2011; Talavage et al., 2014).

The current study reports the cumulative number, average-to-date, and previous week's events for the Facemask, Top-Front, Side, and Back helmet locations and aggregate (Global). Helmet hits above $60 \mathrm{G}(60+\mathrm{G}$ hits) were considered high magnitude as this level represents the 90th percentile of all hits on athletes observed during the period of study (Breedlove et al., 2012).

\section{MR Spectroscopy}

MRS was conducted on a 3T Signa HDx (General Electric; Waukesha, WI) using a 16channel brain array (Nova Medical; Wilmington, MA). Single voxel spectra were collected in the left dorsolateral prefrontal cortex (DLPFC) and dominant primary 
motor cortex (M1) of the athletes (PRESS sequence; 8-mL volume; TR/TE = 1500/30 ms; 128 averages). LCModel (Provencher, 1993), AFNI, and FSL were used to calculate water- and relaxation-adjusted metabolite concentrations. Data with poor quality spectra or where voxel placement was inconsistent with the pre-season scans were discarded, leaving 39 in-season observations for DLPFC and 44 for M1. Additional details on ROI selection, data collection, and procedures may be found in Poole et al. (2014).

\section{Head Collision History}

HITS telemetry data along with month of play were incorporated in a step-wise regression model-per Breedlove et al. (2012)—to identify relationships to deviations from baseline in MRS-measured metabolic concentrations (calculated as In-season - Preseason). Significant predictors for each model were forwarded to a mixed model, adjusted for team and year of data collection, with subject treated as a repeated variable with compound symmetric variance. Statistical Analysis System (Version 9.3) was used to do all statistical analyses and p-values $<0.05$ were considered significant.

\section{$\underline{\text { Results }}$}

Within DLPFC, the mixed model revealed negative correlations between changes in total creatine $(\mathrm{tCr})$ and inositol (Ins) metabolic concentrations and the number of $60+G$ hits experienced in the preceding week. Hit metrics were also predictive for 
both negative and positive changes in $\mathrm{N}$-acetyl aspartate (NAA) and total choline (tCho).

In M1, changes in $\mathrm{tCr}$ and combined glutamate and glutamine (Glx) were predicted by hit metrics. Though M1 Glx had month of assessment (i.e., current duration of exposure) as the strongest predictor, it was also negatively correlated with the percentage of $60+\mathrm{G}$ hits to date. $\mathrm{M} 1 \mathrm{tCr}$ changes were positively correlated to the total number of hits experienced in the previous week.

All hit versus metabolites results, including those from the step-wise regression models, are listed in Figure 1A. An example scatter plot with line of best fit depicting the significant relationship between changes in M1 Glx and percent $60+\mathrm{G}$ hits to date may be found in Figure 1B.

\section{Discussion}

This analysis of metabolism and head collision event history in football athletes expands on the findings of Poole et al. (2014), which suggested damaged glial cells, membrane turnover, and disrupted energy metabolism and neurotransmission in an undiagnosed injured population. Two key findings are drawn from the present work: (1) some metabolites deviant at the group-level for this population were, in fact, correlated with the hits experienced, and (2) other metabolites of interest, not necessarily found to be deviant at the group level, had trends related to the magnitude, number and location of helmet impacts sustained. 
First, DLPFC Ins, DLPFC tCr, and M1 Glx - all concentrations that decreased over the competition season in Poole et al. (2014) - were reported only to correlate negatively with high magnitude hit metrics. Changes in the two DLPFC metabolites correlated with the number of high magnitude hits experienced during the previous week. This suggests that large hits lead to impaired energetics and glial cell damage in a region central to the working memory and executive network. Changes in M1 Glx were correlated with the percent of high magnitude hits on a to-date basis. These three negative relationships with high magnitude hits suggest that large hits induce acute state of hypermetabolism in these areas (Schuhmann et al., 2003).

Second, other metabolites found to have no apparent or variable change at the group-level, had both uni-directional and competing bi-directional relationships with hit metrics. DLPFC NAA, for example, typically reported in clinical studies to decrease following head injury, was found to decrease with increasing to-date and recent high magnitude hits. Although these decreases mirrored other metabolites, NAA increases were also observed with the percentage of high magnitude and overall increasing numbers of global helmet hits. Though increases in NAA are not typically associated with pathology, they have previously been reported in football players who experienced multiple TBIs (Johnson et al., 2012) and warrant further investigation. DLPFC tCho also had conflicting relationships with the type of hits received. While increases were correlated with high numbers of proximal hits likely indicative of membrane synthesis and turnover following brain injury (Zeisel et al., 1991) - transient reductions related to recent high magnitude and global hits 
during the time other metabolites were in low concentration is suggestive of demyelination associated with exposure to direct mechanical stress. It should also be noted that hit relationships with $\mathrm{tCr}$ differed by region: though a negative correlation was found in DLPFC and is presumably reflective of an energy crisis following injury (S. Signoretti, Vagnozzi, Tavazzi, \& Lazzarino, 2010), a positive correlation with the number of hits experienced globally was found with $\mathrm{M} 1 \mathrm{tCr}$, which perhaps reflecting a separate response, also common to injury, for this region (Yeo et al., 2011).

\section{Conclusions}

By monitoring the head insults accrued over time, two mechanisms were revealed in executive and motor neural network brain regions: initial hypermetabolic responses and successive, sometimes opposite, hypometabolic responses, all dependent upon the timing, number, magnitude, and location of blows to the helmet. With diverse metabolic consequences to accumulating sub-concussive blows, such competing mechanisms could (1) lead to no noticeable differences in overall metabolic levels and (2) ultimately mask symptoms in injured athletes. These findings provide evidence for a cumulative effect of head blows on neural health, even though past efforts have failed to pinpoint injuries to a single event.

Future work will aim to address the limitations of a limited sample size, helmet impact characteristics, as well as the possibility that there exist additional nonlinear relationships with the metabolites. 


\section{Acknowledgements}

The authors would like to thank Dr. Gregory G. Tamer, Jr. for assistance in data collection and Dr. Jeffrey W. Gilger for support with statistical analysis methodology. This work was supported by the Indiana Spinal Cord and Brain Injury Research Fund, a part of the Indiana State Department of Health [to TMT, LJL, EAN]. 


\section{References}

Breedlove, E. L., Robinson, M., Talavage, T. M., Morigaki, K. E., Yoruk, U., O'Keefe, K., . . . Nauman, E. A. (2012). Biomechanical correlates of symptomatic and asymptomatic neurophysiological impairment in high school football. $J$ Biomech, 45(7), 1265-1272.

Broglio, S. P., Schnebel, B., Sosnoff, J. J., Shin, S., Fend, X., He, X., \& Zimmerman, J. (2010). Biomechanical properties of concussions in high school football. Med Sci Sports Exerc, 42(11), 2064-2071.

Crisco, J. J., Wilcox, B. J., Beckwith, J. G., Chu, J. J., Duhaime, A. C., Rowson, S., . . . Greenwald, R. M. (2011). Head impact exposure in collegiate football players. J Biomech, 44(15), 2673-2678.

Johnson, B., Gay, M., Zhang, K., Neuberger, T., Horovitz, S. G., Hallett, M., . . . Slobounov, S. (2012). The Use of Magnetic Resonance Spectroscopy in the Subacute Evaluation of Athletes Recovering from Single and Multiple Mild Traumatic Brain Injury. J Neurotrauma.

May, K. H., Marshall, D. L., Burns, T. G., Popoli, D. M., \& Polikandriotis, J. A. (2014). Pediatric Sports Specific Return to Play Guidelines following Concussion. International journal of sports physical therapy, 9(2), 242.

Poole, V. N., Abbas, K., Shenk, T. E., Breedlove, E. L., Breedlove, K. M., Robinson, M. E., ... Dydak, U. (2014). MR spectroscopic evidence of brain injury in the nondiagnosed collision sport athlete. Dev Neuropsychol, 39(6), 459-473.

Provencher, S. W. (1993). Estimation of metabolite concentrations from localized in vivo proton NMR spectra. Magn Reson Med, 30(6), 672-679. 
Schuhmann, M. U., Stiller, D., Skardelly, M., Bernarding, J., Klinge, P. M., Samii, A., . . Brinker, T. (2003). Metabolic changes in the vicinity of brain contusions: a proton magnetic resonance spectroscopy and histology study. J Neurotrauma, 20(8), 725-743.

Signoretti, S., Tavazzi, B., Lazzarino, G., \& Vagnozzi, R. (2014). The Relevance of Assessing Cerebral Metabolic Recovery for a Safe Return to Play Following Concussion Concussions in Athletics (pp. 89-112): Springer.

Signoretti, S., Vagnozzi, R., Tavazzi, B., \& Lazzarino, G. (2010). Biochemical and neurochemical sequelae following mild traumatic brain injury: summary of experimental data and clinical implications. Neurosurg Focus, 29(5), E1.

Talavage, T. M., Nauman, E., Breedlove, E. L., Yoruk, U., Dye, A. E., Morigaki, K., . . Leverenz, L. J. (2014). Functionally-Detected Cognitive Impairment in High School Football Players Without Clinically-Diagnosed Concussion. J Neurotrauma, 31(4), 327-338.

Vagnozzi, R., Signoretti, S., Tavazzi, B., Floris, R., Ludovici, A., Marziali, S., . . . Lazzarino, G. (2008). Temporal window of metabolic brain vulnerability to concussion: a pilot $1 \mathrm{H}$-magnetic resonance spectroscopic study in concussed athletes--part III. Neurosurgery, 62(6), 1286-1295; discussion 1295-1286.

Yeo, R. A., Gasparovic, C., Merideth, F., Ruhl, D., Doezema, D., \& Mayer, A. R. (2011). A longitudinal proton magnetic resonance spectroscopy study of mild traumatic brain injury. J Neurotrauma, 28(1), 1-11. 
Zeisel, S. H., Da Costa, K., Franklin, P. D., Alexander, E. A., Lamont, J., Sheard, N., \& Beiser, A. (1991). Choline, an essential nutrient for humans. The FASEB journal, 5(7), 2093-2098. 
Figure: Relations of metabolite changes to hit history, assessed using the Head Impact Telemetry System (HITS; Simbex, LLC). (A) Table depicting results of mixed model analyses, with those hit metrics exhibiting significant correlations with observed changes in metabolite concentrations highlighted in grey. (B) Example of a statistically significant $(p<0.044)$ regression plot, illustrating changes in M1 Glx as a function of percentage of high magnitude $(60+\mathrm{G})$ hits to-date.

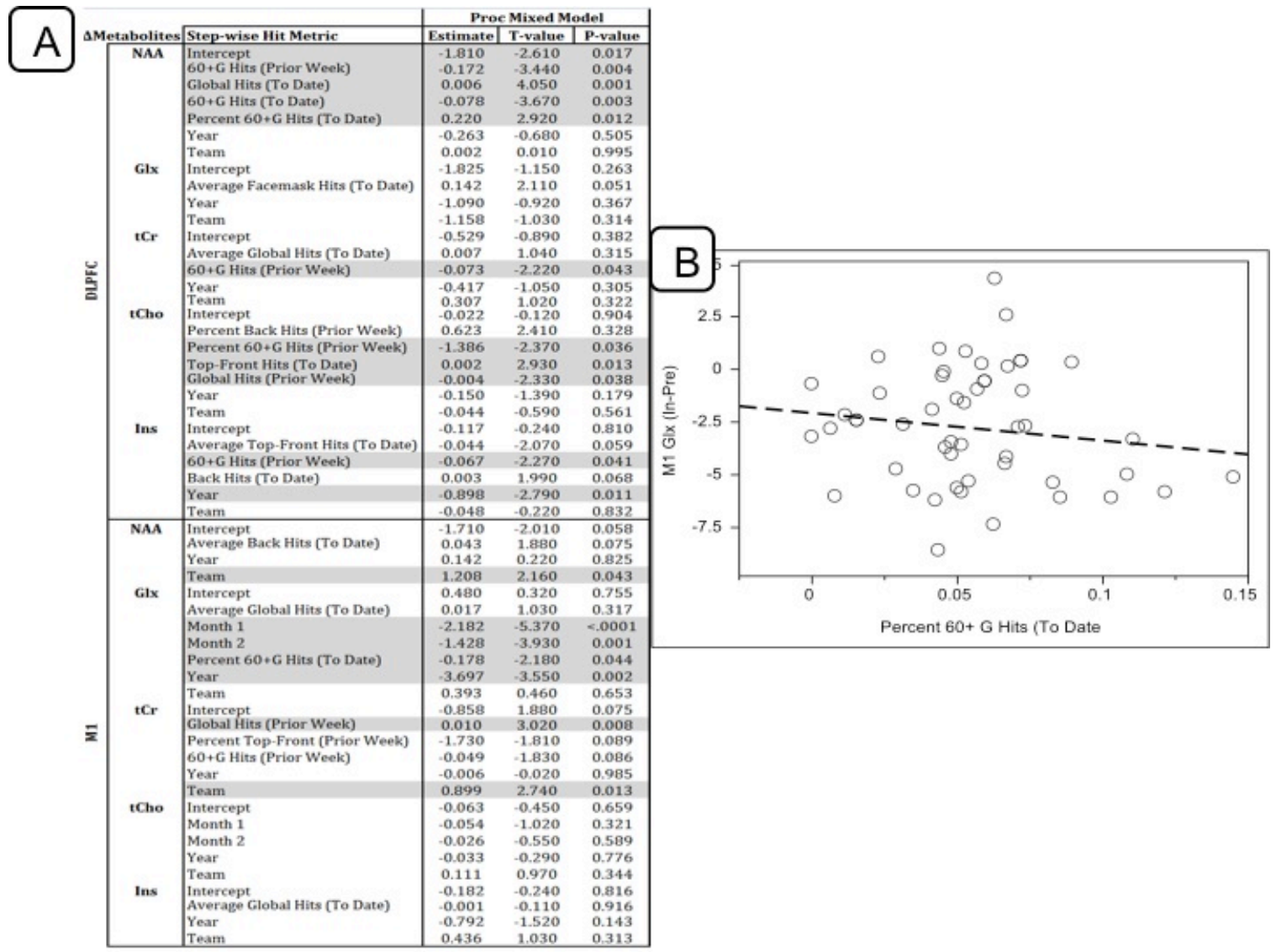


Supplemental Material: Hit metric summaries by season and team.

\begin{tabular}{|c|c|c|c|c|c|c|c|}
\hline & \multirow[b]{3}{*}{ Hit Metric } & \multicolumn{4}{|c|}{ PNG Season 3} & \multirow{2}{*}{\multicolumn{2}{|c|}{$\begin{array}{c}\text { PNG Season } 4 \\
\text { Team } 1(n=11)\end{array}$}} \\
\hline & & \multicolumn{2}{|c|}{ Team $1(n=9)$} & \multicolumn{2}{|c|}{ Team $2(n=15)$} & & \\
\hline & & Mean & Std Dev & Mean & Std Dev & Mean & Std Dev \\
\hline \multirow{3}{*}{$\begin{array}{l}\text { ज़ } \\
\text { 은 }\end{array}$} & Global Hits (Prior Week) & 41.5 & 26.1 & 40.1 & 36.4 & 69.2 & 35.2 \\
\hline & Average Global Hits (To Date) & 35.7 & 13.5 & 36.0 & 17.4 & 66.9 & 26.8 \\
\hline & Total To Date Hits (To Date) & 280.3 & 140.1 & 351.5 & 258.5 & 454.1 & 324.2 \\
\hline \multirow{5}{*}{$\begin{array}{l}\text { U } \\
+ \\
\text { t }\end{array}$} & $60+$ G Hits (Prior Week) & 1.3 & 1.7 & 3.1 & 4.7 & 3.7 & $\overline{2.9}$ \\
\hline & Average $60+\mathrm{G}$ Hits (To Date) & 1.2 & 1.0 & 2.5 & 2.2 & 3.2 & 1.9 \\
\hline & $60+\mathrm{G}$ Hits (To Date) & 9.3 & 7.5 & 24.0 & 22.6 & 23.5 & 19.5 \\
\hline & Percent $60+$ G Hits (Prior Week) & $4 \%$ & $5 \%$ & $6 \%$ & $6 \%$ & $5 \%$ & $4 \%$ \\
\hline & Percent $60+\mathrm{G}$ Hits (To Date) & $4 \%$ & $3 \%$ & $7 \%$ & $3 \%$ & $4 \%$ & $2 \%$ \\
\hline \multirow{5}{*}{ 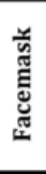 } & Facemask Hits (Prior Week) & 12.5 & 10.8 & 10.4 & 12.1 & 16.4 & 13.2 \\
\hline & Facemask Hits (To Date) & 80.4 & 47.7 & 99.1 & 89.9 & 107.1 & 102.9 \\
\hline & Average Facemask Hits (To Date) & 10.2 & 4.9 & 9.7 & 5.7 & 15.1 & 9.1 \\
\hline & Percent Facemask Hits (Prior Week) & $30 \%$ & $12 \%$ & $24 \%$ & $9 \%$ & $22 \%$ & $11 \%$ \\
\hline & Percent Facemask Hits (To Date) & $29 \%$ & $8 \%$ & $26 \%$ & $7 \%$ & $23 \%$ & $9 \%$ \\
\hline \multirow{5}{*}{ 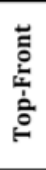 } & Top-Front Hits (Prior Week) & 8.4 & 9.1 & 8.4 & 10.3 & 18.5 & 17.7 \\
\hline & Top-Front Hits (To Date) & 52.1 & 39.0 & 64.9 & 72.1 & 117.3 & 118.9 \\
\hline & Average To Date Top-Front(To Date) & 6.6 & 3.7 & 6.4 & 4.5 & 18.0 & 14.8 \\
\hline & Percent Top-Front (Prior Week) & $17 \%$ & $9 \%$ & $16 \%$ & $10 \%$ & $23 \%$ & $13 \%$ \\
\hline & Percent TopFront Hits (To Date) & $18 \%$ & $7 \%$ & $17 \%$ & $7 \%$ & $24 \%$ & $13 \%$ \\
\hline \multirow{5}{*}{ ڤँ } & Side Hits (Prior Week) & 10.6 & 5.8 & 10.8 & 9.1 & 15.5 & 10.4 \\
\hline & Side Hits (To Date) & 67.5 & 31.7 & 87.6 & 62.7 & 100.1 & 82.4 \\
\hline & Average Side Hits (To Date) & 8.7 & 4.2 & 9.2 & 4.7 & 14.4 & 6.4 \\
\hline & Percent Side Hits (Prior Week) & $28 \%$ & $8 \%$ & $30 \%$ & $14 \%$ & $24 \%$ & $13 \%$ \\
\hline & Percent Side Hits (To Date) & $24 \%$ & $5 \%$ & $26 \%$ & $6 \%$ & $24 \%$ & $10 \%$ \\
\hline \multirow{5}{*}{ 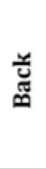 } & Back Hits (Prior Week) & 10.1 & 7.8 & 10.6 & 7.4 & 18.8 & 11.0 \\
\hline & To Date Back Hits (To Date) & 80.3 & 46.7 & 99.4 & 62.7 & 129.6 & 89.1 \\
\hline & Average Back Hits (To Date) & 10.2 & 4.8 & 10.6 & 5.7 & 19.4 & 11.2 \\
\hline & Percent Back Hits (Prior Week) & $25 \%$ & $14 \%$ & $29 \%$ & $10 \%$ & $30 \%$ & $16 \%$ \\
\hline & Percent Back Hits (To Date) & $29 \%$ & $10 \%$ & $30 \%$ & $8 \%$ & $30 \%$ & $12 \%$ \\
\hline
\end{tabular}

